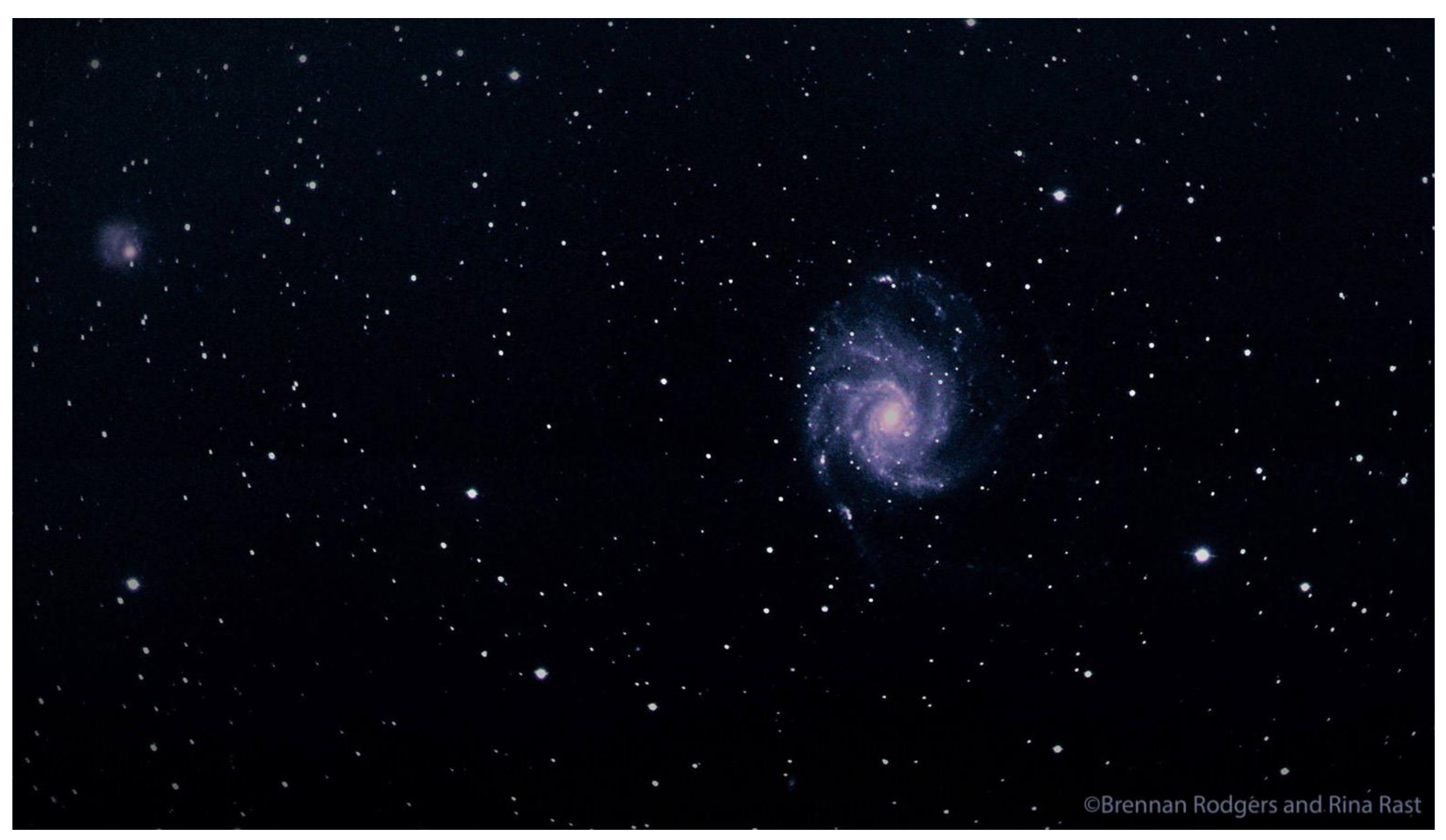

\title{
Cover Image
}

Pinwheel Galaxy

Rina Rast and Brennan Rodgers, with assistance from Emery Dlugan

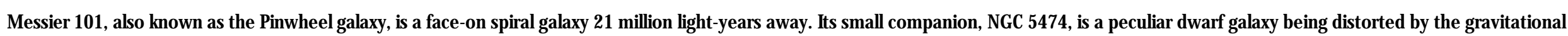
influence of the Pinwheel. This is a stacked image of 30x180 second exposures using an 11" RASA telescope and colour CM OS camera on the roof of the USask Physics Building.

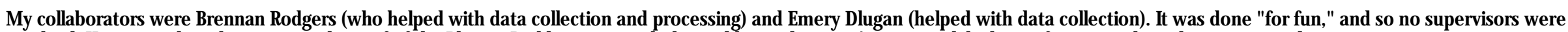
involved. However, the telescopes on the roof of the Physics Building are regularly used by students in Astronomy lab classes for research in photometry and spectroscopy. 